\title{
Barriers to male involvement in uptake of Prevention-of-Mother- to-Child-Transmission (PMTCT) of HIV in Sub-Saharan Africa
}

\author{
"Rebecca John Osman, RM, RN, HND, BAM, PDRH, *Regine Unkels, MD, MPH,

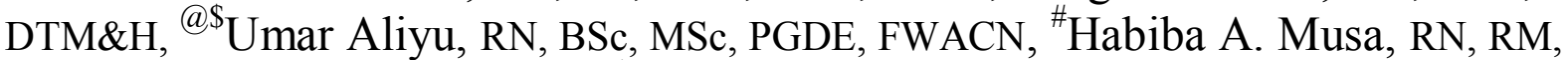 \\ A\&E, ADPA, FWACN and ${ }^{\$}$ Otonoku K. Mathew, RN, RNA, BNSc, MSc \\ ${ }^{\#}$ Gwarinpa General Hospital, Life Camp, P.M.B. 206, Health \& Human Services Secretariats FCDA, Garki- \\ Abuja \\ *Centre for Maternal and Newborn Health, Department of International Public Health, Liverpool School of \\ Tropical Medicine \\ ${ }^{\S}$ Department of Nursing Services, National Hospital, Abuja
}

\begin{abstract}
Sub-Saharan Africa continues to bear the burden of HIV/AIDS in the world, with countries like Ethiopia, Congo, Eritrea and Nigeria constituting the highest percentages of the burden. Women and children are the mostly affected. Nigeria was reported second on the list of countries with the highest number of People living with HIV/AIDS (PLWHA) which was 3.5 million in 2011 including pregnant women. Despite the fact that prevention of mother-to-child-transmission of HIV/AIDS (PMTCT) services are provided free in Sub-Saharan Africa, research shows inadequate uptake. This study identifies reasons for low uptake of PMTCT associated to male involvement and provides evidence from the literature on best practices to overcome low male involvement. A literature search was conducted and 22 articles were selected for this review. Findings revealed that if men participated in PMTCT programmes and supported their wives, uptake could increase. Important barriers to male involvement identified were fear of stigmatization and the belief that men should not participate in female reproductive health.
\end{abstract}

Key words: Male barriers, involvement, participation, transmission, HIV

\section{Introduction}

Haile, et al. (2014) reported that many children are infected with HIV by their mothers, "90\% of childhood infection were from mother-to-child-transmission and about 600.000 children are infected annually", despite increased emphasis on advocacy and awareness creation, provision of health care facilities and availability of intervention measures such as free HIV counselling and testing, provision and supply of antiretroviral drugs.

Donor agencies, both local and international, have contributed significant funds to strengthen PMTCT services and uptake worldwide yet the results presented in respect to mother-to-child-transmission are not encouraging (Brusamento, et al. 2012). This impediment calls for a careful needs assessment in order to identify problems at different levels and address them individually.

For every 10 men that got infected with HIV in 2011, 13 women were infected and about 23.5 million people were living with HIV in Sub-Saharan Africa, approximately 57\% of which are women (UNAIDS 2012). These represented $69 \%$ of the total global HIV burden and $92 \%$ of the pregnant women living with HIV globally in 2011. Only 59\% of them are reached by Prevention of Mother-to-Child-Transmission (PMTCT) programmes. In addition, more than $90 \%$ of the children who acquired HIV in 2011 are from Sub-Saharan Africa. Countries that had PMTCT coverage of less than 25\% are: Angola, Chad, Congo, Eritrea, Ethiopia, Nigeria and South Sudan (Folayan 2004) (figure 2). Nigeria has an HIV prevalence of 4.I\% among pregnant women but only $10 \%$ of these are receiving antiretroviral prophylaxis (figure1, figures 3) (UNAIDS 2012).

"It's a man's world" is a cliché that is often used to allude to the gender disparity favouring men. This is especially evident in developing countries like Nigeria where women are regarded as the lesser sex not deserving equal societal privileges. Men have a lot of power attached to their status in the family and the wider society. However, these attributes have become part of the barriers to male involvement in the uptake of PMTCT and low utilization (Mbonye et al. 2010).

The supremacy concept surrounding male gender has negative effects such that the roles of men have not been sufficiently considered important in PMTCT planning (Sherr, et al., 2012). Many men are not involved in the ANC/PMTCT process because years of gender power imbalance have created a culture which fences out men from female reproductive health, considered a woman's issue which should be handled by women alone (Gupta 2002). 


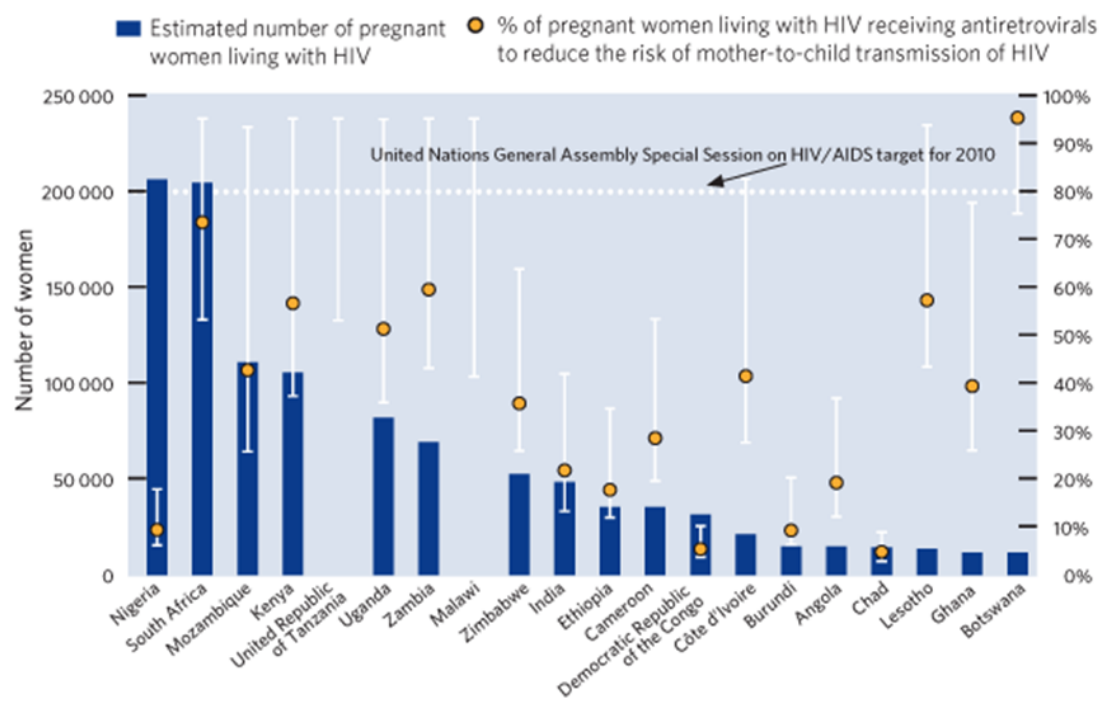

1 The bar indicates the uncertainty range around the estimate.

Source: World Health Organization (WHO, 2008)

Figure 1: Showing percentage of pregnant women receiving anti-retroviral prophylaxis

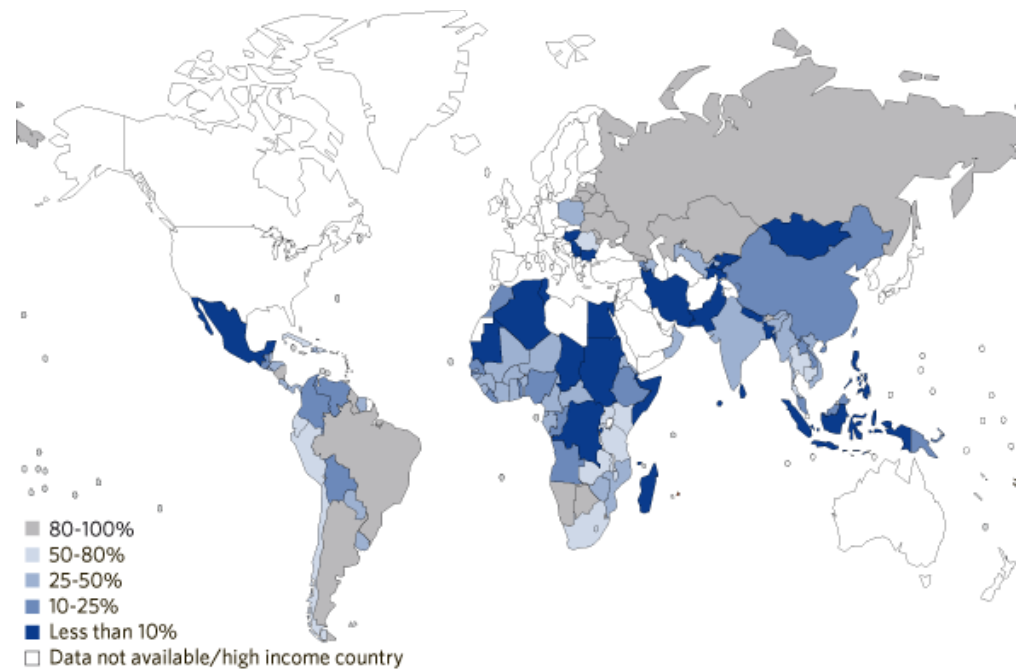

Source: http://who.int/hiv/topics/mtet/mtct graph 6.gif

Figure 2: Coverage of Antiretroviral to PMTCT $<25 \%$

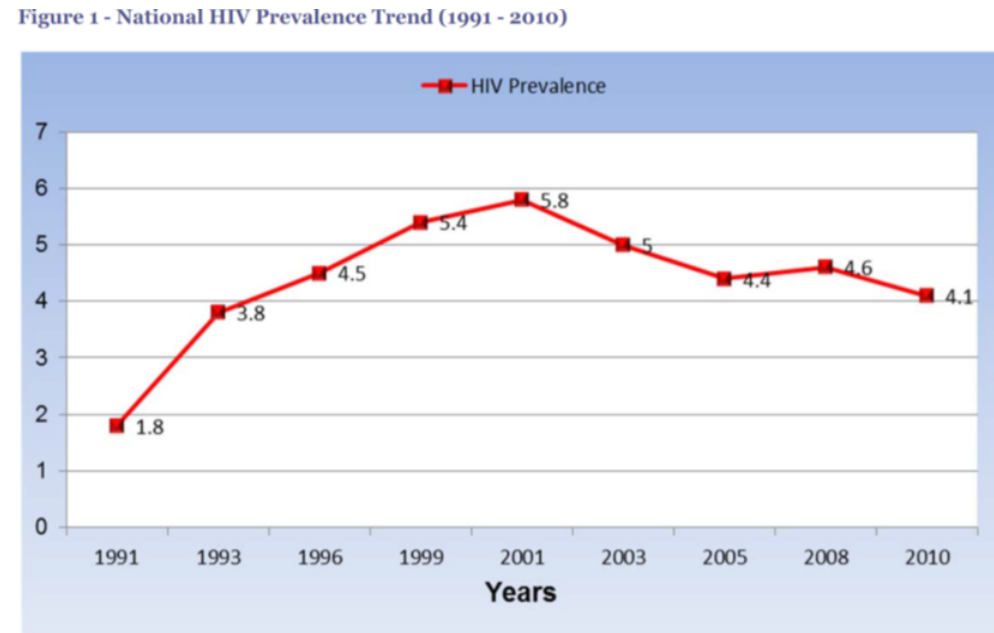

Source: National Agency for Control of Aids (2013)

Figure 3: Nigeria HIV Prevalence Trend in (1991-2010) 


\section{Methods}

This article is based on the project report for the award of the Diploma in Sexual and Reproductive Health at Liverpool School of Tropical Medicine (LSTM), undertaken by Rebecca John Osman. The literature review was carried out using the following data bases: PubMed, Embase and the Cochrane library. The key word search in these databases generated 42 studies among which 22 articles were carefully studied to generate this piece of work.

\section{Key words used in database search}

Male barriers AND involvement OR participation AND PMTCT AND Sub-Saharan Africa

\section{Study question}

What are the barriers causing low male involvement in PMTCT programmes in Sub-Saharan Africa and what are best practices to overcome these?

\section{Objectives}

- To identify the major barriers hindering men from participating in ANC/PMTCT services with their wives in Sub-Saharan Africa

- To identify best practices to increase male involvement in PMTCT programmes

\section{Barriers to male involvement}

\section{Results}

Madiba, et al. (2013), discovered that disclosure of HIV sero-status to a partner is always the main gate to progress in PMTCT uptake and that is what pregnant women in Sub-Saharan Africa do not have confidence to do, due to the fear of negative reactions they will face from their husbands and relations. A study carried out by Bajunirwe et al (2005) in rural and urban Uganda found that women refused HIV testing in antenatal clinic due to fear of their husbands' reactions.

King et al. (2008), reported from PMTCT programmes in Uganda that most of the women who were interviewed did not actually experience any negative outcome after disclosure rather the fear of the disclosure itself created high tension in them, which almost became a barrier to sharing a positive HIV result with partners.

However, Medley et al. (2004) reported that in South Africa the rate of women who experienced violence, discrimination, abandonment and accusation of infidelity after disclosure of their HIV result ranges from 3.5\% to 14.6\%. Akarro, et al. (2011), Ditekemena, et al. (2012), Koo, et al. (2013) and Taylor, et al. (2013) all found the concept in their studies, that men have negative assumptions about antenatal care. They believe it is a women's forum and so what should a man be found doing there. According to their findings a man who accompanies his wife to ANC/PMTCT and is seen by other men will be subjected to stigmatization through his peers, e.g. they may say his wife dominates him or that his power has been taken away from him and he is not worthy to be a man.

Thus, men are faced with difficult cultural challenges and that can be a major reason why they shy away from involvement in PMTCT programmes as well. Sarker et al. (2007) also attributed low uptake of PMTCT and failure of women to return for their HIV results to poor partner's support. In addition, men are also afraid of testing for HIV for fear of a positive result. As Getu (2011) reported a particular male respondent talked about the mental torment of testing for HIV saying 'it will be too much for me to bear, so I will never go for an HIV test on my own unless other circumstances led to it'. Also lack of awareness of what PMTCT is and its importance to the well-being of families may be another reason for low male involvement in PMTCT uptake in Sub-Saharan Africa (Brusamento et al. 2012).

\section{Best practice to increase male involvement}

Ditekemena, et al. (2012) and Koo, et al. (2013) report that mothers that adhered to PMTCT programmes are more often accompanied by their husbands to ANC/PMTCT clinic than those who do not adhere. They also found that these couples have some level of education and found it easier to freely discuss issues related to their HIV status and family health care management. Therefore these studies show that awareness and communication between the couples will likely make men participate more in ANC/PMTCT programmes.

An interventional trial among pregnant women in Kenya showed increased uptake of voluntary counselling and testing (VCT) and promotion of safe sex during pregnancy if men were invited to PMTCT (Mohlala, et al., 2011). In addition, one study in Kenya emphasized a positive correlation between male involvement and infant outcome (Aluisio et al. 2011). According to Mbonye et al. (2010) the participation of men not only improves mothers attendance in PMTCT but also enhanced utilization of facility delivery. 
Peltzer, et al. (2011) stated that media campaigns improved male participation in prevention of HIV programmes in Botswana from 4\%-11\%, while a study in Zambia used financial incentives for any couple that attended HIV counselling together which increased male involvement in PMTCT and in Tanzania, since men became involved, the uptake of Nevirapine increased (in mothers 55 vs $24 \%$. $\mathrm{p}=0.07$ and infant 55 vs $22 \%$. $\mathrm{P}=0.06$ ) (Pletzer et al 2011). This research shows that if partners are involved the goals of PMTCT programmes can be achieved.

Haile, et al. (2014) documented in Ethiopia that only 95 male partners out of 473 accompanied their partners to ANC/PMTCT even though out of these $82 \%$ got tested and counselled for HIV. Male participation will provide support for the woman (Ramirez-Ferrero, et al., 2012) and may therefore enhancing the uptake and utilization of PMTCT. King et al. (2008) reported from a project in Uganda that male VCT and PMTCT uptake increased after special invitations were sent to male partners.

\section{Barriers to male involvement}

\section{Discussion}

In an attempt to find answers to the problems with partner disclosure of positive HIV result King et al. (2008); Madiba, et al., (2013) and Medley et al. (2004) conducted in-depth interviews and focus group discussions (FGDs) with pregnant women who tested HIV positive from ANC and with their male partners. The outcome of the interviews with the women revealed fear of their husbands' reaction and insecurity how to tell them about the results. Men mentioned fear as one of the barriers that prevented them from participating in ANC/PMTCT. They feared the reactions of their wives to the positive result and were also afraid of stigmatisation which is also found by Turan, et al., (2013). Discussions among those couples could have alleviated the fears of both partners. Ezeanolue, et al. (2013) found that most of the women interviewed for their study, said they would be more comfortable to share their HIV positive result with their husbands if they were tested together on the same day.

Some of the barriers affecting the uptake and involvement of men in PMTCT in Sub-Saharan Africa that were identified through the literature review are as follows:

\section{Fear of disclosure to partners}

Akarro, et al. (2011) and Koo, et al. (2013) reported that fear is a big factor that stops pregnant women that tested HIV positive from revealing their status to their husbands and thus involve them in their care. They fear losing their marriages or relationships and be abandoned with their babies. Disclosure can be made easy if proper counselling is done. Medley, et al. (2004) reported that pregnant women who cannot tell their partners, are encouraged to allow the nurses or counsellors to disclose to their partners since they have the skill do it better and in a more neutral way at the same time providing correct facts about the disease and its management. A similar approach can be adopted in other countries in Sub-Saharan Africa and a change in policy should be advocated to allow for different models of disclosure according to the clients wishes.

\section{Lack of awareness}

Brusamento et al. (2012), explained that most PMTCT awareness efforts have been directed almost exclusively at women, oblivious to the cultural role men play in women's decision-making. They further assert that this situation has led men to admitting that they do not have sufficient knowledge or understanding of their roles in the prevention of HIV. Acceptance of any concept relies on how well this concept is understood, so if husbands have a clear understanding of PMTCT they can be enabled to make an informed decision that will benefit their family. Involving men in PMTCT ANC will also lead to an increased uptake of facility delivery which was mentioned before with a propable impact on maternal and newborn mortality. Integration of HIVand reproductive health services will lead to increased cost-effectiveness and synergies and even more so if men are routinely involved. HIV-testing in PMTCT can be an entry point to this type of male involvement.

\section{Socio-cultural factors and stigmatisation}

According to Reece (2010), the perception of male gender inequality is a barrier to male involvement in PMTCT. Men believed that culturally ANC/PMTCT is a women's activity and so a man should not be seen there. When a man accompanies his wife and he is seen by other men they may stigmatize him. Gender-based stigma has been identified as a barrier to reproductive health services for women in the literature as decisionmaking and the generation of funds for treatment of women is still left to men. Increased involvement of men in reproductive care may lead to better utilization of health care by women or even increased decision-making power for women regarding their own health. 


\section{Attitudes of service providers}

Byamugisha et al. (2010) documented in a cross sectional survey in Uganda, the rudeness and hostility sometimes experienced by men from health providers. Men described the overly aggressive nature of physical examinations of their wives. In many instances, the men were not allowed to enter the ANC clinics with their wives even if they wanted to. Ditekemena, et al. (2012) confirm this finding, stating that the harsh treatment meted out to the men "discouraged them from returning or participating in PMTCT activities".

\section{Economic factors}

According to the study done by Nkuoh et al. (2010) men complained of ANC and obstetric care bills as an obstacle hindering them from participating in ANC/PMTCT. They indicated the overbearing demands of their wives for money for one ANC activity or the other. In addition accompanying the wife to hospital brings about waste of time which will not afford them the opportunity to go about looking for their means of livelihood. ANC and also PMTCT services are officially free of charge in almost all Sub-Saharan African countries. However, in reality, unofficial, indirect and opportunity cost sum up to a significant expenditure for these services which may deter many couples from attending. Governments and also health facility management need to make sure that maternity services are delivered free of charge and that access to them is equitable.

\section{Best practice to increase male involvement}

The methods adopted by TASO Jinja in Uganda as reported by King et al. (2008) helped to alleviate barriers associated to fear of disclosure to partners by sending special invitations to male partners, which increased VCT and uptake of PMTCT. Peltzer, et al. (2011) stated that media campaigns can improve male participation in prevention of HIV programmes as shown in Botswana as well as financial incentives for any couple that attends HIV counselling. One best practice from Nigeria shows very promising results but hasn't been properly evaluated yet.

In Gwarinpa General Hospital in Abuja every woman that comes with her husband to register in ANC will be attended to early. In his personal communication by email Momoh (2014) described the procedure at Gwarinpa General Hospital as follows: midwives, the medical director, pharmacist and an assigned laboratory scientist give health talks on topics like HIV pre-test counselling and other topics related to pregnancy and childbirth. HIV testing is started immediately after health education in the counselling unit, result are disclosed to the couples together by counsellors and those who are positive have post- test counselling again, as they are immediately registered on the PMTCT programme.

An interesting lesson learnt from this method is that the couples' minds are already prepared for the test outcome before the result is out due to specific information on the procedure during health education, so whatever is the outcome they are positioned together to work towards fighting the disease since they have been taught there is hope of not dying immediately and that if they adhere to the programme the likelihood of transmission to their child will be small. The second lesson, most importantly to the couple is the benefit of revealing all information to each other and it makes them support one another (Homsy et al., 2006). In his personal communication by email Momoh (2014) reported that this practice has increase the involvement of men in ANC/PMTCT uptake and optimal utilization of the services. It may also improve treatment adherence of both partners and decrease loss to follow up of mother-and-child pairs.

\section{Implications for policy and practice}

1. Stigma reduction to increase male participation in ANC but also in HIV testing.

This is a concept that is difficult to change but creating awareness and basic education will help in societal and individual behavioural change, (Piot et al. 2008).

\section{Reduction of fear of disclosure}

Encourage couple HIV counselling and testing together Testing together will make the couple support each other and decrease fear of how to present the results to the partner. The counsellors too can be authorised by the clients to disclose results were problems are feared.

3. Awareness creation

Efforts in creating awareness should be directed at couples rather than just the women. This could take the form of jingles, billboards, handbills or other education resources such as radio and TV spots, but also as demonstrated in Nigeria, health education for couples.

4. Change in provider attitude: Health workers should be trained on attitude modification already at pre-service training and any service provider caught being rude to clients should be warned and if not change penalised. Such events often occur when the workers are tired due to work overload and understaffing, Therefore there should be more staff on duty especially on most busy days in departments with a high turn-over of clients and where staff is dealing with sensitive issues such as 
HIV-testing and counselling. Duty allocation should be clear and concise so that staff responsibilities will be evident to the provider and the client.

\section{Conclusions}

Fear to disclose a positive HIV test result to partners, fear of testing, stigmatization influenced by sociocultural factors, long waiting time in the hospital leading to decreased income generation thereby affecting family income and attitude of health workers were identified as the main barriers to male involvement in PMTCT in our review.

In our opinion, awareness creation for men and women on what a PMTCT programme is and its importance to the family will resolve the problem of disclosure and stigmatization together with couple counselling and different models of disclosure. Advocacy to governments and all key stakeholders to provide an enabling environment is also fundamental to the uptake of PMTCT. If all these are put in place, men may be more willing to support their wives by participating in ANC/PMTCT.

\section{References}

[1]. Akarro, R.R., Deonisia, M. \& Sichona, F. (2011) 'An Evaluation of Male Involvement on the Programme for PMTCT of HIV/AIDS: A Case Study of Ilala Municipality in Dar es Salaam, Tanzania', Arts \& Social Sciences Journal, vol. 2011.

[2]. Aluisio, A., Richardson, B.A., Bosire, R., John-Stewart, G., Mbori-Ngacha, D. \& Farquhar, C. (2011) 'Male antenatal attendance and HIV testing are associated with decreased infant HIV infection and increased HIV free survival', Journal of acquired immune deficiency syndromes (1999), vol. 56, no. 1, p. 76.

[3]. Bajunirwe, F. \& Muzoora, M. (2005) 'Barriers to the implementation of programs for the prevention of mother-to-child transmission of HIV: a cross-sectional survey in rural and urban Uganda', AIDS Research and Therapy, vol 2, no 10

[4]. Brusamento, S., Ghanotakis, E., Tudor Car, L., van-Velthoven, M.H., Majeed, A. \& Car, J. (2012) 'Male involvement for increasing the effectiveness of prevention of mother-to-child HIV transmission (PMTCT) programmes', Cochrane Database Syst Rev, vol. 10, p. Cd009468.

[5]. Byamugisha, R., Tumwine, J.K., Semiyaga, N. \& Tylleskär, T. (2010) 'Research Determinants of male involvement in the prevention of mother-to-child transmission of HIV programme in Eastern Uganda: a cross-sectional survey'.

[6]. Ditekemena, J., Koole, O., Engmann, C., Matendo, R., Tshefu, A., Ryder, R. \& Colebunders, R. (2012) 'Determinants of male involvement in maternal and child health services in sub-Saharan Africa: a review', Reproductive health, vol. 9, no. 1, p. 32.

[7]. Ezeanolue, E.E., Obiefune, M.C., Yang, W., Obaro, S.K., Ezeanolue, C.O. \& Ogedegbe, G.G. (2013) 'Comparative effectiveness of congregation-versus clinic-based approach to prevention of mother-to-child HIV transmission: study protocol for a cluster randomized controlled trial', Implementation Science, vol. 8, no. 1, p. 62.

[8]. Folayan, M. (2004) 'HIV/AIDS: the Nigerian response', The Political Economy of AIDS in Africa, pp. 85-104.

[9]. Getu, D.K. (2011) 'Factors related to male participation in prevention of mother-to-child transmission of human immunodeficiency virus in three public hospitals in Addis Ababa, Ethiopia'.

[10]. Gupta, G.R. (2002) 'How men's power over women fuels the HIV epidemic: It limits women's ability to control sexual interactions', BMJ: British Medical Journal, vol. 324, no. 7331, p. 183.

[11]. Haile, F. \& Brhan, Y. (2014) 'Male partner involvments in PMTCT: a cross sectional study, Mekelle, Northern Ethiopia', BMC Pregnancy and childbirth, vol. 14, no. 65 .

[12]. Homsy, J., Kalamya, J.N., Obonyo, J., Ojwang, J., Mugumya, R., Opio, C. \& Mermin, J. (2006) 'Routine intrapartum HIV counseling and testing for prevention of mother-to-child transmission of HIV in a rural Ugandan hospital', JAIDS Journal of Acquired Immune Deficiency Syndromes, vol. 42, no. 2, pp. 149-154.

[13]. King, R., Katuntu, D., Lifshay, J., packel, L., Patamwita, R., Nakayiwa, S., Abang, B., Babirye, F., Lindkvist, P., Johansson, E., Mermin, J. \& Bunnell, R. (2008) 'Process and outcomes of HIV serostatus disclosure to sextual partners among people living with HIV in Uganda', AIDS and Behavior, vol. 12, pp. 232-243.

[14]. Koo, K., Makin, J.D. \& Forsyth, B.W. (2013) 'Barriers to Male-Partner Participation in Programs to Prevent Mother-to-Child HIV Transmission in South Africa', AIDS Education and Prevention, vol. 25, no. 1, pp. 14-24.

[15]. Madiba, S. \& Letsoalo, R. (2013) 'HIV disclosure to partners and family among women enrolled in prevention of mother to child transmission of HIV program: implications for infant feeding in poor resourced communities in South Africa', Glob J Health Sci, vol. 5, no. 4, pp. 1-13.

[16]. Mbonye, A., Hansen, K., Wamono, F. \& Magnussen, P. (2010) 'Barriers to prevention of mother-to-child transmission of HIV services in Uganda', Journal of biosocial science, vol. 42, no. 2, p. 271.

[17]. Medley, A., Garcia-Moreno, C., McGill, S. \& Maman, S. (2004) 'Rates, barriers and outcomes of HIV serostatus disclosure among women in developing countries: implications for prevention of mother-to-child transmission programmes', Bull World Health Organ, vol. 82, no. 4, pp. 299-307.

[18]. Mohlala, B.K., Boily, M.-C. \& Gregson, S. (2011) 'The forgotten half of the equation: randomized controlled trial of a male invitation to attend couple voluntary counselling and testing', AIDS (London, England), vol. 25, no. 12, p. 1535.

[19]. Momoh, A. A. (2014) Personal communication by email. Unpublished.

[20]. Nkuoh, G.N., Meyer, D.J., Tih, P.M. \& Nkfusai, J. (2010) 'Barriers to Men's Participation in Antenatal and Prevention of Mother-to-Child HIV Transmission Care in Cameroon, Africa', Journal of Midwifery \& Women's Health, vol. 55, no. 4, pp. 363369.

[21]. Peltzer, K., Jones, D., Weiss, S.M. \& Shikwane, E. (2011) 'Promoting male involvement to improve PMTCT uptake and reduce antenatal HIV infection: a cluster randomized controlled trial protocol', BMC public health, vol. 11, no. 1, p. 778.

[22]. Piot, P.,Bartos, M., Larson, H., Zewdie, D. \& Mane, P . (2008). Coming to terms with complexity: A call to action for HIV Prevention, Lancet 372 (9641), 845 - 859

[23]. Ramirez-Ferrero, E. \& Lusti-Narasimhan, M. (2012) 'The role of men as partners and fathers in the prevention of mother-to-child transmission of HIV and in the promotion of sexual and reproductive health', Reproductive health matters, vol. 20, no. 39, pp. 103109. 
[24]. Sarker, M., Sanou, A., Snow, R., Ganame, J. \& Gondos, A. (2007) 'Determinants of HIV counselling and testing participation in a prevention of mother-to-child transmission programme in rural Burkina Faso', Tropical Medicine \& International Health, vol. 12, no. 12 , pp. 1475-1483.

[25]. Sherr, L. \& Croome, N. (2012) 'Involving fathers in prevention of mother to child transmission initiatives-what the evidence suggests', Journal of the International AIDS Society, vol. 15, no. Suppl 2.

[26]. Turan, J.M. \& Nyblade, L. (2013) 'HIV-related stigma as a barrier to achievement of global PMTCT and maternal health goals: a review of the evidence', AIDS and Behavior, vol. 17, no. 7, pp. 2528-2539.

[27]. UNAIDS (2012) Global Report Factsheet [Online], UNAIDS, Available from: http://www.unaids.org/documents/20101123 FS SSA em en.pdf (Accessed: 9 March 2014).

[28]. WHO (2013) HIV in the WHO African Region: Progress towards achieving Universal Access to priority health sector interventions, vol., [Online]. Available from: http://www.afro.who.int/en/clusters-a-programmes/dpc/acquired-immune-deficiencysyndrome/features/3950-hiv-in-the-who-african-region-progress-towards-achieving-universal-access-to-priority-health-sectorinterventions-2013-update.html (Accessed 2014). 